\title{
Three-dimensional ultrastructural imaging reveals the nanoscale architecture of mammalian cells
}

Shengkun Yao ${ }^{1}$, jiadong Fan $^{2}$, Zhiyun Chen ${ }^{3}$, Yunbing Zong ${ }^{4}$, Jianhua Zhang ${ }^{5}$, Zhibin Sun ${ }^{5}$, Lijuan Zhang ${ }^{6}$, Renzhong Tai ${ }^{7}$, Zhi Liu ${ }^{5}$, Chunying Chen ${ }^{8}$ and Huaidong Jiang ${ }^{5}$

${ }^{1}$ Shandong Normal University, United States, ${ }^{2}$ ShanghaiTech University, United States, ${ }^{3}$ National Center for Nanoscience and Technology of China, China (People's Republic), ${ }^{4}$ Shandong University, China (People's Republic), ${ }_{5}^{5}$ hanghaiTech University, China (People's Republic), ${ }^{6}$ Shanghai Synchrotron Radiation Facility, Shanghai Institute of Applied Physics, Chinese Academy of Science, China (People's Republic), ${ }^{7}$ Shanghai Synchrotron Radiation Facility, Shanghai Institute of Applied Physics, Chinese Academy of Science, United States, ${ }^{8}$ National Center for Nanoscience and Technology of China, United States

Nanotechnology has dramatically increased humans' intended and unintended exposure to nanomaterials, which generate various biological effects. Some nanomaterials exhibit diagnostic and therapeutic effects for certain diseases in medical applications, whereas others can cause possible adverse effects. These diverse effects are determined by the interactions between nanomaterials and biological systems, peculiarly at the subcellular level, including cellular uptake, intracellular localization and translocation. Imaging techniques that can visualize nanomaterials within whole cells at nanometer resolution now play a crucial role in nanomedicine and nanotoxicology.

To explore the interactions between nanomaterials and cells, dual-energy X-ray microscopy is a promising choice and can achieve quantitative nondestructive three-dimensional (3D) imaging of both cellular structures and nanomaterials at nanometer resolution simultaneously. Dual-energy contrast X-ray microscopy is based on the abrupt change in the absorption when the energy of the incident X-rays changes from just below the absorption edge of a specific element to above it. The observed differences in images collected at two energies reflect the locations of element-specific nanomaterials. This method can simultaneously image the ultrastructures of cells and intracellular nanomaterials and, thus, can be used to investigate the interactions between nanomaterials and biological systems.

We applied dual-energy contrast X-ray microscopy to explore the intracellular localization of nanomaterials in whole large-size mammalian cells. By combing scanning transmission X-ray microscopy (STXM) and a Fourier-based iterative tomographic algorithm termed equally sloped tomography (EST), we investigated the quantitative 3D subcellular distribution of $\mathrm{Gd} @ \mathrm{C} 82(\mathrm{OH}) 22$ within a macrophage. Large numbers of nanoparticles (NPs) aggregated within the cell and were mainly located in phagosomes. No NPs were observed in the nucleus. Imaging of the nanomedicine within whole cells advanced our understanding of the highefficiency antitumor activities and low toxicity of this agent. This imaging technique can be used to probe nanomaterials within intact large-size cells at nanometer resolution uniformly in three dimensions and may greatly benefit the fields of nanomedicine and nanotoxicology. 
A

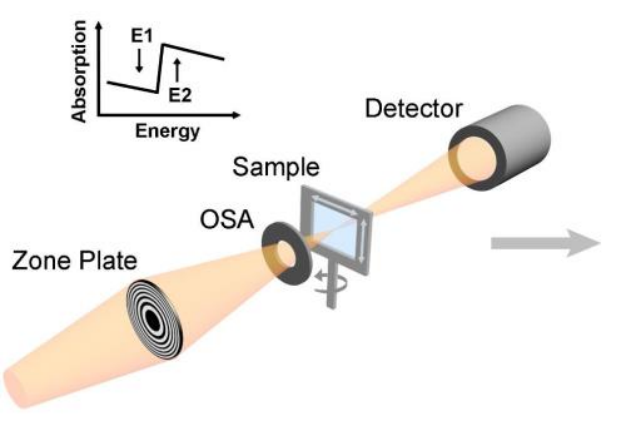

B

E1

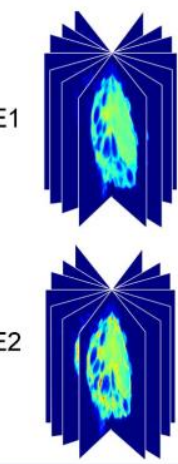

C

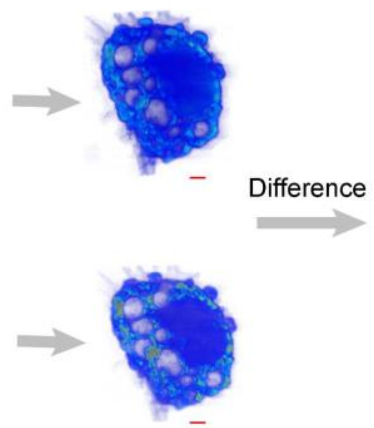

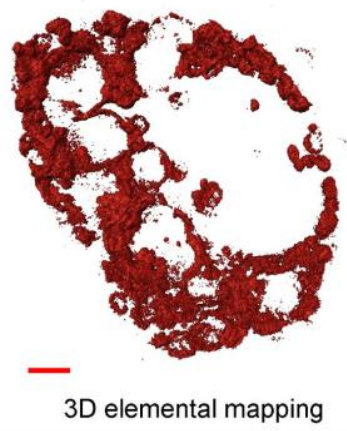

Figure 1. Fig 1. Schematic layout of the dual-energy image technique. (A) Two sets of projections were acquired by STXM at energies below and above the absorption edge of a specific element; (B) the projections are reconstructed by the EST algorithm separately; and (C) the quantitative 3D distribution of the specific element was calculated based on the abrupt change in the absorption. 
A

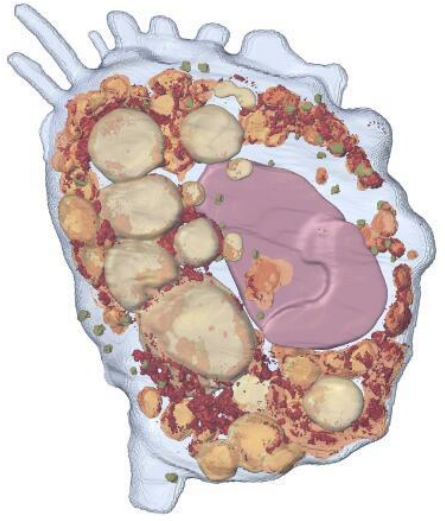

C

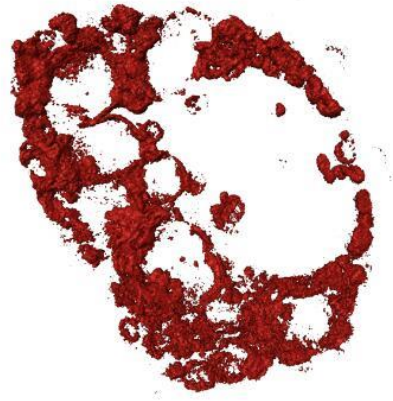

3D elemental mapping
B
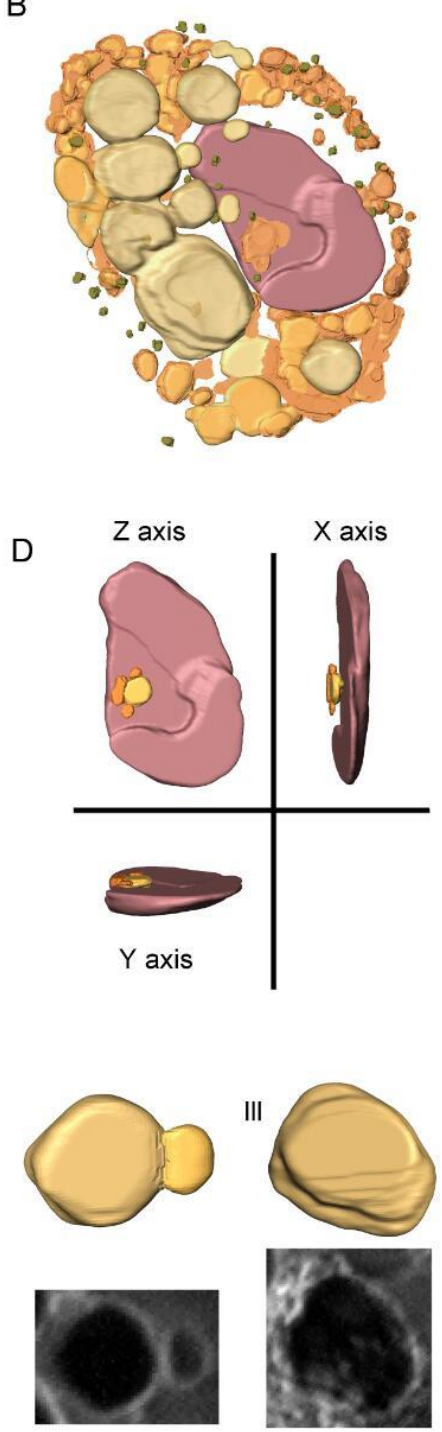
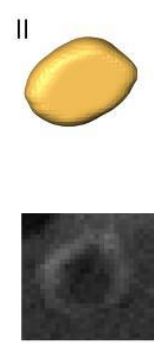

Figure 2. Fig 2. The intracellular distribution of the nanomaterials. (A) Volume rendering of the reconstructed macrophage showing [Gd@ $\mathrm{C} 82(\mathrm{OH}) 22] n$ (dark red), nucleus (brown), and different types of lysosomes (yellow). (B) Organelle segmentation based on differences in the linear absorption and specific morphology. (C) 3D distribution of [Gd@C82(OH)22]n, showing that the NPs aggregated and exhibited a characteristic distribution in the cell. (D) Zoomed-in view of the nucleus in three dimensions showing that the NPs were distributed in four separated lysosomes on the surface of the nucleus. (E) Three different types of lysosomes were distinguished based on the density and distribution of [Gd@C82(OH)22]n, which may reflect the intracellular transportation of the nanomaterials.

\section{References}

1. Yao, Shengkun, et al. "Three-dimensional ultrastructural imaging reveals the nanoscale architecture of mammalian cells." IUCrJ 5.2 (2018): 141-149.

2. Jiang, Huaidong, et al. "Quantitative 3D imaging of whole, unstained cells by using X-ray diffraction microscopy." Proceedings of the National Academy of Sciences 107.25 (2010): 11234-11239.

3. Miao, Jianwei, Friedrich Förster, and Ofer Levi. "Equally sloped tomography with oversampling reconstruction." Physical Review B 72.5 (2005): 052103. 
4. The following funding is acknowledged: the National Natural Science Foundation of China (12004227 , 31430031, 11425520, 11290165), Shandong Provincial Natural Science Foundation of China (ZR2020QA076), and the Major State Basic Research Development Program of China (grant No. 2014CB910401). 